\title{
Expression of microRNAs miR-126 and miR-873 and genes CASPASE-8 and C-FLIP in neurospheres of Glioblastoma lines U-343 subjected to treatment with ionizing radiation and temozolomide.
}

\author{
F. Pansani ${ }^{1}$, L.P. Turra ${ }^{1}$, F.S. Lizarte Neto ${ }^{1}$, M.L. de A. Cirino ${ }^{1}$, \\ L.F. Tirapelli ${ }^{2}$, F.M. Peria ${ }^{2}$, C.G. Carlotti Junior ${ }^{1}$ and \\ D.P. da C. Tirapelli ${ }^{1}$ \\ ${ }^{1}$ Departamento de Cirurgia e Anatomia, Faculdade de Medicina de Ribeirão \\ Preto, Universidade de São Paulo, Ribeirão Preto, SP, Brasil \\ ${ }^{2}$ Departamento de Medicina Interna, Faculdade de Medicina de Ribeirão Preto, \\ Universidade de São Paulo, Ribeirão Preto, SP, Brasil \\ Corresponding author: D.P. da C. Tirapelli \\ E-mail: lab.biomol.cirurgia@fmrp.usp.br
}

Genet. Mol. Res. 18 (4): gmr18261

Received February 11, 2019

Accepted October 16, 2019

Published November 30, 2019

DOI http://dx.doi.org/10.4238/gmr18261

\begin{abstract}
Glioblastoma is considered incurable, even with a combination of therapies (chemo and radiotherapy), and surgical resection. New therapeutic approaches are needed to improve the prognosis of patients with glioblastoma. In recent decades, research has focused on molecular biology of brain tumors. We examined the role of programmed cell death, apoptosis, through two microRNAs that act as possible pro and anti-apoptotic gene regulators. We evaluated the expression of the genes CASPASE-8 and C-FLIP and microRNAs miR126-5p and miR-873-5p in adhered cells (AC) and neurospheres (NS) from cell line U343, which were submitted to temozolomide (TMZ) and ionizing radiation (IR), isolated and associated (TMZ + IR). We concluded that microRNA-126 and 873 were differentially expressed as as a function of treatment regime in glioblastomas. The higher expression of the caspase- 8 gene in adhered cells in the group treated with IR when compared to the other groups at time $48 \mathrm{~h}$ suggests that the ionizing radiation in the adhered cells of the glioblastoma cell line culture has apoptosis-inducing properties.
\end{abstract}

Key words: Glioblastoma; Apoptosis; miRNA; Gene 


\section{INTRODUCTION}

Gliomas account for one-third of all brain tumors of the central nervous system, and also account for $80 \%$ of malignant primary tumors of the brain and CNS (Wohrer et al., 2009; Ostrom et al., 2017; Darlix et al., 2017). Glioblastoma multiforme (GBM) is considered incurable and with high aggressiveness, even with a combination of therapies, such as maximum surgical resection followed by adjuvant radiotherapy (RT) with simultaneous treatment and adjuvant temozolomide (TMZ) (Stupp et al., 2009). Radiotherapy followed by adjuvant temozolomide remains the standard therapy for newly diagnosed GBM (Lieberman, 2017).

The diagnosis of GBM requires some histological criteria, such as hypercellularity, nuclear atypia and mitotic activity, in addition to microvascular proliferation and/or tumor necrosis (Aldape et al., 2015).

Diffuse gliomas occur more frequently in adults and are the more common intrinsic brain tumors that present wide range of clinical behaviors, and may be of slow clinical progression in patients with grade II tumors up to the mean of short survival in patients with grade IV tumors (GBM) of 12-18 months (Aldape et al., 2015).

Mean overall survival increases to 14.6 months with the advent of TMZ during and after radiotherapy compared with isolated radiotherapy with the ability to differentiate the effects of newly diagnosed tumor therapy and progression, neuroimaging is being an innovative approach (Lieberman, 2017).

Because glioblastoma is a highly aggressivene tumor, new more effective therapeutic strategies are needed. Studies on the molecular genetics of GBM are underway to understand the molecular pathways that influence resistance to immunotherapy and other control measures (Dunn et al., 2012; Stupack, 2013; Lieberman, 2017).

The expression of Caspase-8 in GBM is often withdrawn, and is upregulated in a mesenchymal group (Verhaak et al., 2010; Stupack, 2013). But the silencing of Caspase-8 can sensitize the cancer cells with the use of the TMZ in the treatment (Fianco et al., 2016; Fianco et al., 2017).

Apoptosis is one of the major cell death pathways, and when it does not, it suggests that its loss of expression or impairment of its apoptotic activation triggers the resistance of cancer cells (Fianco et al., 2018; Humphreys, 2018). Therefore studies on apoptosis are important because understanding its mechanisms, it improves the therapies used in the treatment of cancer (Fulda, 2015; Pfeffer and Singh, 2018).

C-FLIP proteins, which modulate the enzymatic activation of Caspase-8, are regulated in several tumors and correlate with clinical worsening, possibly associated with the inhibitory function of C-FLIP in Caspase-8 (Humphreys, 2018). Tumors that have shown lower Caspase-8 expression or higher levels of C-FLIP may be resistant to the therapies, since the expression of caspase- 8 or proteins that modulate its function may be associated with radiotherapy and chemotherapy, increasing tumor apoptosis (Stupack, 2013; Safa et al., 2011; Humphreys, 2018).

The microRNAs are small non-coding RNAs, containing from 18 to 25 nucleotides, single strand that regulate gene expression by directing mRNA transcripts, which lead to its repression or translational degradation, are regulators of gene expression. They demonstrate complex results, as a single miRNA has the ability to simultaneously regulate multiple genes (Zoni et al., 2015; Tang et al., 2016). 
The targets of the microRNAs correspond to $30 \%$ of all genes encoding proteins. As the miRNAs have several functions, among them, control of cell differentiation, proliferation and apoptosis; aberrant expression and dysregulation contribute to tumorigenesis, angiogenesis and metastasis, and may serve as tumor suppressor or oncogenes (Li et al., 2013)

In relation to apoptosis, there are two routes: intrinsic pathway also known as mitochondrial, triggered by intracellular stimuli (DNA damage, treatment with cytotoxic drugs, lack of growth factors and / or oxidative stress); this pathway is dependent on the formation of apoptosome (compound of pro-caspase-9) activation factor of apoptotic protease 1 (Apaf-1) (Verbrugge et al., 2010; Zhenyi et al., 2015; Aslan et al., 2018).

On the other hand, the extrinsic pathway of apoptosis is initiated by the ligands of death (ligand Faz, TRAIL, TNF- $\alpha$ ), then this interaction, followed by the assembly of the death inducer signaling complex (DISC) containing Fas protein FADD) and procaspase- 8 and procaspase-10. Thus, caspases-3/6/7 induce the end of the cell or end up cleaving Bcl-2, Bid to form the tBid which thus trigger the intrinsic apoptosis pathway that is mediated by mitochondria (Verbrugge et al., 2010; Zhenyi et al., 2015; Aslan et al., 2018).

MiR-126 is associated with various types of tumor. Liu et al., 2014, analyzed that this microRNA functions as a tumor suppressor in colorectal cancer (Liu et al., 2014). Jiang et al. (2014) concluded that miR-126 inhibits cell growth, invasion and migration of osteosarcoma cells.

Zhang et al. (2016) concluded in their study an association of miR-126 with CRC progression and metastasis, thus suggesting that this microRNA may function as a tumor suppressor involved in the development of CRC, being a potential biomarker in the diagnosis and therapeutic act.

Wang et al. (2015) studied miR-873 being linked in the development of GBM. Therefore, they found that the low level of miR-873 was associated with carcinogenesis and metastasis via the promotion of the IGF2BP1 protein. They concluded that miR-873 was down-regulated in GBM cell lines and tissues; which was directly bound to the IGF2BP1 protein through the 3'-UTR binding site; and, finally, that ectopic expression of miR-873 inhibited the proliferation and invasion of GBM cell lines, providing strong evidence that this microRNA acts as a tumor suppressor in the development of GBM, and may implicate miR-873 as a potential target for GBM therapies.

Schulte et al. (2011) analyzed a subset of GBM stem cell lines, the tumoral cell-like glioblastoma (GSf) phenotype, as it has all the criteria for a glioma stem cell and the human GBM transcriptome closer whereas other lineages. Thus, Fiscon et al. (2018) made an analysis comparing genes in $15 \mathrm{GSf}$ cell lines and original tumors in relation to conventional GBM cell lines.

Different expressions of miRNAs have been described in cancer stem cells (CSCs) of individual tumors, suggesting specific functions of the tumor cell type. In GBM, there are onco-miR clusters that influence survival, thus highlighting the effects that microRNAs have on tumor heterogeneity (Huang et al., 2016).

The aim of this study was to evaluate the gene expression of caspase-8, C-FLIP and the expression of miRNAs miR-126-5p and miR-873-5p in neurospheres and adhered cells in glioblastoma cell lines submitted to treatment with temozolomide and with ionizing radiation, isolated and associated. 


\section{MATERIAL AND METHODS}

\section{Cell culture}

The cell lines were originally obtained from the American Type Culture Collection (ATCC). Cells were cultured and maintained at $37^{\circ} \mathrm{C}$ with $5 \%$ carbon dioxide in Dulbelcco's modified Eagle medium (DMEM) containing 10\% (v/v) fetal bovine serum (FBS), $100 \mathrm{IU} / \mathrm{mL}$ penicillin, $100 \mu \mathrm{g} / \mathrm{mL}$ streptomycin/neomycin and $1 \%$ nonessential amino acids (Invitrogen).

\section{Treatment of cells}

The cell cultures were divided into four groups of treatment: an untreated group, a group IR: treated with ionizing radiation (x-ray $6 \mathrm{mV}$ produced by the Siemens linear accelerator with a dose rate of $2.0 \mathrm{~Gy} / \mathrm{min}$ at doses of 6,10 and 14Gy), a group TMZ: treatment with Temozolomide (the compound was diluted to $10 \mathrm{mmol} / \mathrm{L}$ using $99 \%$ DMSO and stored at $-20^{\circ} \mathrm{C}$, was obtained from Sigma ${ }^{\circledR}$ Chemical Co., St. Louis, MO, USA and Schering-Plough Brazil) and a group treatment with the association of both treatment: IR+TMZ. All groups were analyzed at $48 \mathrm{~h}$.

\section{RNA isolation and real-time polymerase chain reaction}

Total RNA was extracted with Trizol reagent (Applied Biossystems, USA) according to the manufacture's instructions. In preparation of real-time polymerase chain reaction (PCR), reverse transcription of RNA samples was performed using the High-Capacity cDNA kit (Applied Biossystems, USA).

Real time PCR: The cDNA was amplified with quantitative Real Time Polymerase Chain Reaction (q-PCR) using TaqMan Master Mix (Applied Biosystems) for reaction.

The U6 gene was used as an endogenous control (housekeeping) for reaction of the microRNA, however for gene reaction TBP and HPRT were used as endogenous controls. The PCR conditions were: pre-heating at $50^{\circ}$ for 2 min, denaturation at $95^{\circ} \mathrm{C}$ for $10 \mathrm{~min}$ and 50 cycles of amplification and quantification $\left(15 \mathrm{sec}\right.$ at $95^{\circ} \mathrm{C}$ and $1 \mathrm{~min}$ at $60^{\circ} \mathrm{C}$ ). All reactions were carried out in duplicate and analyzed with the 7500 Sequence Detection System apparatus (Applied Biosystems). The data were analyzed using the ABI-7500 SDS software. Dissociation curves were performed (melting curves) after amplification by RQ-PCR. The samples that showed dissociation curves with different temperature and/or more than one point of dissociation in the same sample were discarded and repeated.

\section{Statistical Analysis}

The statistics and data analysis was performed using ABI prism and GraphPadPrism 5 software. 


\section{RESULTS}

Exposure to TMZ did not result in modification of the expression of CAPS-8 in adhered cells and neurospheres between each other and in relation to the control group. We also observed an increase in the expression of caspase- 8 in adhered cells in the IR group (Figure 1).

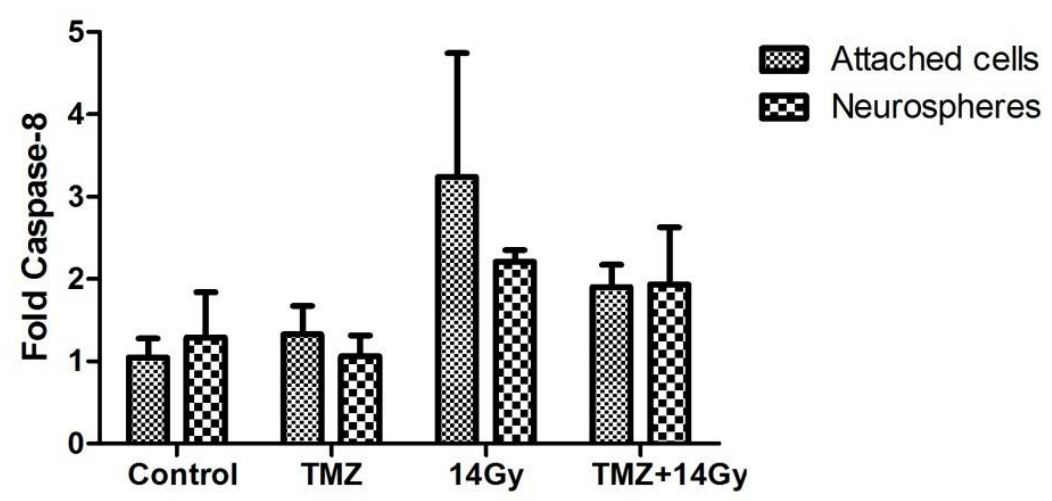

Figure 1. Expression of the CASPASE- 8 gene in the U343 line in the control group and in the groups submitted to the treatment modalities (TMZ, IR, TMZ + IR) in the studied subgroups: neurospheres and attached cells.

Intervention with IR produced significant C-FLIP expression in adhered cells and neurospheres, when compared to the TMZ + IR treatment group. We also observed a decrease in C-FLIP expression in the two groups treated with TMZ + IR when compared to the groups of adhered cells treated with IR (Figure 2).

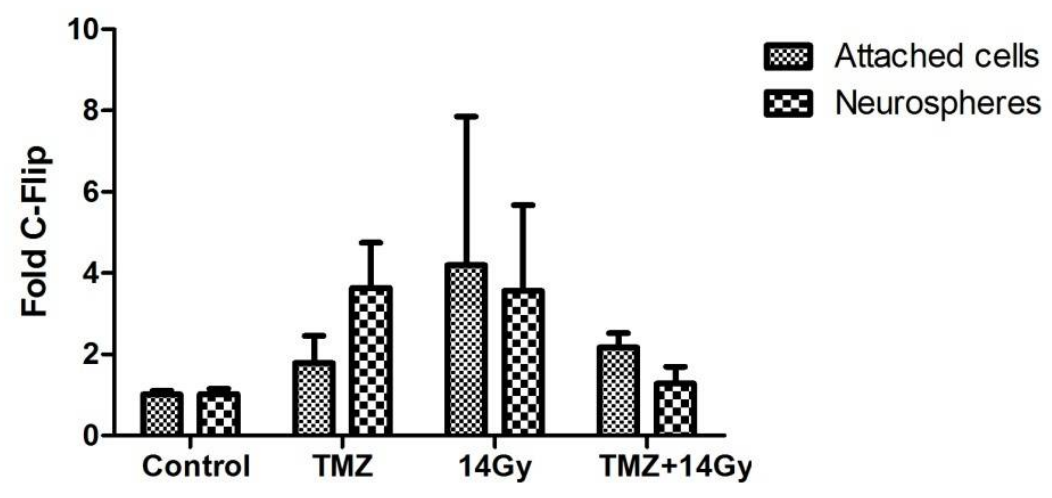

Figure 2.- Expression of the C-FLIP gene in the U343 line in the control group and in the groups submitted to the treatment modalities (TMZ, IR, TMZ + IR) in the studied subgroups: neurospheres and attached cells.

Exposure to TMZ alone resulted in significant expression of miR-126 in adhered cells. We observed a decrease in miR-126 expression in neurospheres when exposed to TMZ (Figure 3). 


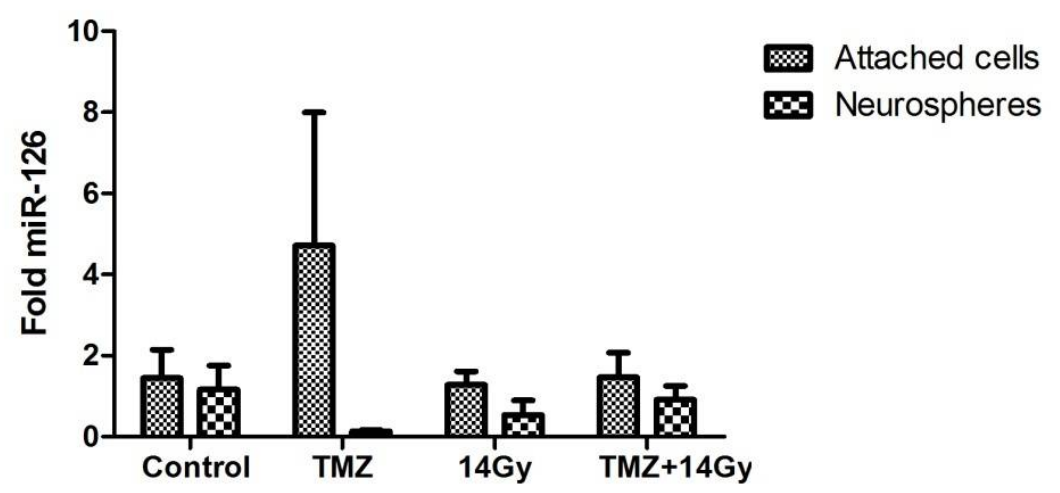

Figure 3. Expression of the miRNA-126 in the U343 line in the control group and in the groups submitted to the treatment modalities (TMZ, IR, TMZ + IR) in the studied subgroups: neurospheres and attached cells.

We observed increased expression of miR-873 in the adhered cells in the TMZ, IR and TMZ + IR groups, when compared to the other groups of adhered cells and neurospheres (Figure 4).

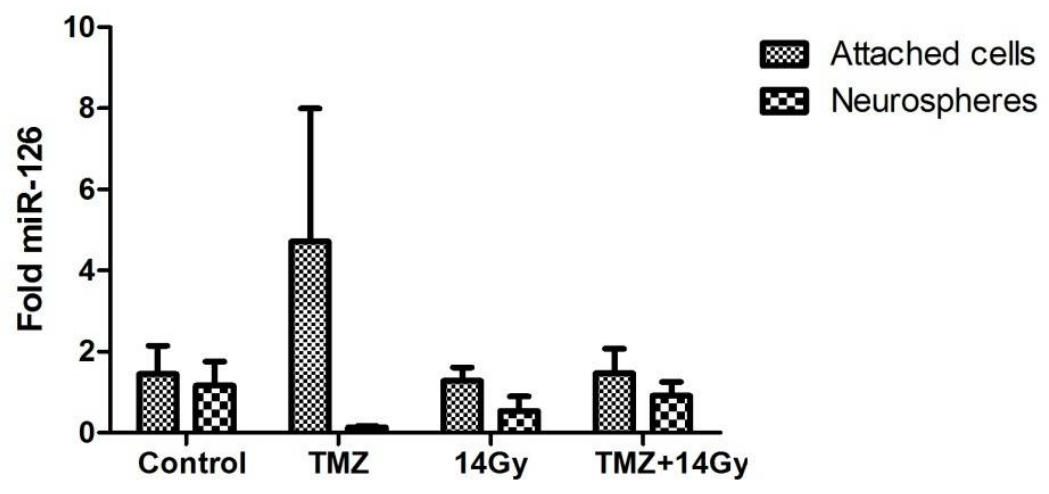

Figure 4. Expression of the miRNA-873 in the U343 line in the control group and in the groups submitted to the treatment modalities (TMZ, IR, TMZ + IR) in the studied subgroups: neurospheres and attached cells.

\section{DISCUSSION}

Studies provide evidence of caspase- 8 expression levels in GBM and primary tumors (Ashely et al., 2005; Kuijlen et al., 2010; Verhaak et al., 2010; Stupack, 2012). Fianco et al. (2016 and 2017), reported that expression of caspase-8 is retained in cell lines, U87MG and U251MG, in GBM. With these models, neoplastic transformation and resistance to TMZ treatment in vitro, supporting the activation of NF-kB, cytokine production, neoangiogenesis and tumor growth in vitro and in vivo.

Verhaak et al. (2016) examined the upregulation of caspase-8 in GBM patients compared to normal tissue, particularly in the mesenchymal subtype. Responsible for the initiation and execution of apoptosis, caspase- 8 is a canonical cysteine protease. Apoptosis, 
also known as induced programmed cell death and its activation is necessary for the functionality of this pathway (Muzio et al., 1996; Juo et al., 1998).

Considered a tumor suppressor because it plays a central role in apoptosis induced by death receptors, it is in agreement with the observation that the expression of Caspase- 8 or its enzymatic activity (necessary for the execution of the apoptotic signal), are impaired in cancer (Juo et al., 1998; Stupack, 2013).

The C-FLIP protein is able to modulate the enzymatic activity of caspase- 8 and modulate recruitment to various signaling pathways, indicating C-FLIP capable of disrupting the functionality of caspase-8 (Fianco et al., 2018).

Many tumors repress caspase- 8 expression, even if their apoptotic activity is impaired by C-FLIP protein levels, so the expression of this protein is regulated abnormally in cancer (Humphreys et al., 2018). Chang and colleagues in 2002 analyzed that the level of C-FLIP expression is very important for the regulation of apoptosis, determining whether it will be activated or not, and is an important therapeutic target with death receptor signaling (Chang et al., 2002).

Boege et al. (2017) suggested that inhibition of caspase-8, which impairs the induction of apoptosis, may increase the sensitivity of cancer cells to agents that damage DNA independently of apoptosis, representing a valuable therapeutic strategy.

The expression of caspase- 8 is enriched in some gliomas, suggesting a complex role not only in the development of cancer, but also in the modulation of chemotherapeutic sensitivity (Verkhaak et al., 2010; Stupack et al., 2013; Fianco et al., 2018).

Modulation of caspase-8 may affect chemotherapy sensitivity not only by interfering with the apoptotic response, but also by additional pathways. Negative regulation of caspase-8 in GBM may trigger resistance to apoptosis, correlates with a reduction in the level of expression of inflammatory cytokines, resulting in increased sensitivity to TMZ via a yet unknown molecular pathway (Fianco et al., 2017).

Meister and colleagues in 2010 analyzed that miR-126 and miR-126* play different roles in processes such as inflammation, blood vessel growth and cancer. Related to cancer and in different types of neoplastic diseases, it inhibits tumor growth through proliferation, migration and invasion (Meister, 2010).

\section{ACKNOWLEDGMENTS}

This study was carried out with the support of the Coordenação de Aperfeiçoamento de Pessoal de Nível Superior - Brasil (CAPES) - Financing Code 001.

\section{CONFLICTS OF INTEREST}

The authors declare no conflict of interest.

\section{REFERENCES}

Aldape K, Zadeh G Mansouri S, et al. (2015). Glioblastoma: pathology, molecular mechanisms and markers. Acta Neuropathol. 129(6): 829-48. 10.1007/s00401-015-1432-1.

Aslan E, Akbas E, Yilmaz S, et al. (2018). Ear atresia: Is there a role of apoptosis-regulating miRNAs? North Clin. Istanb. 5(3): 238-245.

Chang DW, Xing Z, Pag Y, et al. (2002). c-FLIP(L) is a dual function regulator for caspase-8 activation and CD95mediated apoptosis. EMBO J. 21:3704-14.

Genetics and Molecular Research 18 (4): gmr18261

OFUNPEC-RP www.funpecrp.com.br 
Darlix A, Zouaoui V, Rigau V, et al. (2017). Epidemiology for primary brain tumors: a nationwide population-based study. J. Neurooncol. 131 (3): 525-546.

Dunn GP, Rinne ML, Wykosky J, et al. (2012). Emerging insights into the molecular and cellular basis of glioblastoma. Genes Dev. 26: 756-784.

Finco G, Cenci C and Barila D (2016). Caspase-8 expression and its Src-dependent phosphorylation on Tyr380 promote cancer cell neoplastic transformation and resistance to anoikis. Exp. Cell Res. 347: 114-122.

Fianco G, Mongiardi MP, Levi A, et al. (2017). Caspase-8 contributes to angiogenesis and chemotherapy resistance in glioblastoma. eLife 6: e22593.

Fiscon G, Conte F and Paci P (2018). Swin tool application to expression data of glioblastoma stem-like cell lines, corresponding primary tumors and conventional glioma cell lines. BMC Bioinformatics. 30: 19 (Suppl 15): 436.

Fulda S (2015). Targeting apoptosis for anti cancer therapy. Semin. Cancer Biol. 31: 84-88.

Humprheys L, Espona-Fiedler M and Longley DB (2018). FLIP as a therapeutic target in cancer. FEBS J.Vol. 285:, No. 22, 11.2018, p. 4104-4123.

Huang T, Alvarez AA, Pangeni RP, et al. (2016). A regulatory circuit of miR-125b/miR-20b and Wnt signalling controls glioblastoma phenotypes through FZD6-modulated pathways. Nat Commun. 7: 12885.

Jiang L, He A, Zhang Q et al. (2014). MiR-126 inhibits cell growth, invasion and migration of osteosarcoma cells by downregulating ADAM-9. Tumour Biol. 35: 12645-12654.

Juo P, Kuo C.J, Yuag J et al. (1998). Essential requirement for Caspase-8/FLICE in the initiation of the Fas-induced apoptotic cascade. Curr. Biol. 8: 1001-1008.

Li D, Iinytskyy Y, Kovalchuk A, et al. (2013). Crucial role for early growth response-1 in the transcriptional regulation of miR-20b in breast cancer. Oncotarget. 4(9): 1373-87. PMID: 23945289.

Lieberman F (2017). Glioblastoma update: molecular biology, diagnosis, treatment, response assessment, and translational clinical trials. F1000 Res. 6: 1892.

Liu Y, Zhou Y, Feng X, et al. (2014). MicroRNA-126 functions as a tumor suppressor in colorectal cancer cells by targeting CXCR4 via the AKT and ERK1/2 signaling pathways. Int J Oncol. 44: 203-210.

Meister J and Schmidt MHH (2010). miR-126 and miR-126*: New Players in cancer. Scientific World Journal. 10: 2090-2100. doi: 10.1100 / tsw.2010.198.

Muzio M, Chinnaivan AM, Kissckel FC, et al. (1996). FLICE, a novel FADD-homologous ICE/CED-3-like protease, is recruited to the CD95 (Fas/APO-1) death-inducing signaling complex. Cell. 85: 817-827.

Ostrom QT, Gittleman H, Liao P, et al. (2017) CBTRUS statistical report: primary brain and other central nervous system tumors diagnosed in the United States in 2010-2014 Neuro Oncol. 19: (suppl_5), pp. v1-v88.

Schutle A, Gunther HS, Philppis HS, et al. (2011) A distinct subset of glioma cell lines with stem cell-like properties reflects the transcriptional phenotype of glioblastomas and overexpresses cxcr4 as therapeutic target. Glia. 59(4): 590-602.

Stupp R, Heigi ME, Mason WP, et al. (2009) Effects of radiotherapy with concomitant and adjuvant temozolomide versus radiotherapy alone on survival in glioblastoma in a randomised phase III study: 5-year analysis of the EORTC-NCIC trial. Lancet Oncol. 10: 459-466.

Stupack D (2013). Caspase-8 as a therapeutic target in cancer. Cancer Lett. 332: 133-140.

Tang Z, Takahashi Y, Chen C et al. (2017). Atg2A/B deficiency switches cytoprotective autophagy to non-canonical Caspase-8 activation and apoptosis. Cell Death Differ. 24: 2127-2138.

Verbrugge I, Jonhstone RW and Smythi MJ (2010). SnapShot: extrinsic apoptosis pathways. Cell. 143: 1192

Verhaak G, Hoadley KA, Purdom E, et al. (2010). Integrated genomic analysis identifies clinically relevant subtypes of glioblastoma characterized by abnormalities in PDGFRA, IDH1, EGFR, and NF1. Cancer Cell 17: 98-110.

Wang RJ, Li JW, Bao BH, et al. (2015). MicroRNA-873 (miRNA-873) inhibits glioblastoma tumorigenesis and metastasis by suppressing the expression of IGF2BP1. J. Biol. Chem. 3: 290(14): 8938-48.

Wohrer A, Waldhor T, Heinzl T, et al. (2009). The Austrian Brain Tumour Registry: a cooperative way to establish a population-based brain tumour registry. J. Neurooncol. 95: 401-411.

Zhang GM, Luo L, et al. (2016). MicroRNA-126 inhibits tumor cell invasion and metastasis by downregulating ROCK1 in renal cell carcinoma. Mol Med Rep. 13(6): 5029-2036.

Zoni E, Van Der Plujim G, Gray PC, et al. (2015). Epithelial plasticity in cancer: unmasking a microRNA network for TGF $\beta$, Notch, and Wnt-mediated EMT. J. Oncol. 2015: 198967. 